\title{
SARS-CoV-2-mediated evasion strategies for antiviral interferon pathways
}

\author{
Soo-Jin Oh and Ok Sarah Shin* \\ BK21 Graduate Program, Department of Biomedical Sciences, \\ College of Medicine, Korea University Guro Hospital, Seoul 08308, \\ Republic of Korea \\ (Received Oct 8, 2021 / Revised Nov 24, 2021 / Accepted Nov 24, 2021)
}

With global expansion of the COVID-19 pandemic and the emergence of new variants, extensive efforts have been made to develop highly effective antiviral drugs and vaccines against SARS-CoV-2. The interactions of coronaviruses with host antiviral interferon pathways ultimately determine successful viral replication and SARS-CoV-2-induced pathogenesis. Innate immune receptors play an essential role in host defense against SARS-CoV-2 via the induction of IFN production and signaling. Here, we summarize the recent advances in innate immune sensing mechanisms of SARS-CoV-2 and various strategies by which SARS-CoV-2 antagonizes antiviral innate immune signaling pathways, with a particular focus on mechanisms utilized by multiple SARS-CoV-2 proteins to evade interferon induction and signaling in host cell. Understanding the underlying immune evasion mechanisms of SARS-CoV-2 is essential for the improvement of vaccines and therapeutic strategies.

Keywords: SARS-CoV-2, COVID-19, interferon, immunity

\section{Introduction}

Severe acute respiratory syndrome coronavirus 2 (SARSCoV-2), the causative agent behind coronavirus disease 2019 (COVID-19), has become one of the gravest public health challenges of the 21st century (Li et al., 2020a; Zhou et al., 2020). SARS-CoV- 2 has rapidly spread across 200 countries worldwide with more than 250 million confirmed cases, including over 5 million deaths as of November 2021, according to World Health Organization (COVID-19) dashboard. COVID-19 patients exhibit broad range of clinical manifestations, ranging from mild symptoms such as headache, dry cough, and flu-like symptoms to severe symptoms such as critical pneumonia, septic shock, multiple organ dysfunction,

*For correspondence. E-mail: oshin@korea.ac.kr; Tel.: +82-2-2626-3280;

Fax: +82-2-2626-1962

Copyright (c) 2022, The Microbiological Society of Korea and even death (Chen et al., 2020b; Huang et al., 2020; Wu and McGoogan, 2020).

Coronaviridae family of viruses contain non-segmented, positive sense single-stranded RNA genome that encodes proteins for viral replication, translation, and virion assembly (Perlman and Netland, 2009). SARS-CoV-2 genome is approximately 30,000 nucleotides in length. Several studies have revealed that SARS-CoV-2 genome shares $82 \%$ and $50 \%$ sequence identity with SARS-CoV and MERS-CoV genomes respectively (Krishnamoorthy et al., 2020; Lu et al., 2020; Zhou et al., 2020). The genome of SARS-CoV-2 encodes 4 structural proteins, 16 non-structural proteins (NSPs) and 6 accessory proteins.

Structural proteins consist of spike (S), envelope (E), membrane $(\mathrm{M})$, and nucleocapsid $(\mathrm{N})$ proteins that are involved in viral pathogenesis, viral RNA genome replication, and virion assembly (Cui et al., 2019; Zhu et al., 2020). Among the structural proteins, $S$ protein interacts with the host cell membrane receptor angiotensin converting enzyme 2 (ACE2) and is essential for viral entry into the host cells. Subsequently, the host protease TMPRSS2 cleaves S protein/ACE2 receptor complex to facilitate complete viral entry into the host cell (Hoffmann et al., 2020). Finally, SARS-CoV-2 gain access to host replication and translation machinery to duplicate and express their genes and proteins respectively.

NSPs are produced as cleavage products of two polyproteins encoded by the open reading frame (ORF) la and ORF1b, which form approximately $70 \%$ of the viral genome from the $5^{\prime}$ end. NSPs play a key role in viral replication and gene expression through the formation of replication and transcription complexes (RTCs). Viral RTCs consist of RNA-dependent RNA polymerase (translated from NSP12), methyltransferases (translated from NSP14 and NSP16) to edit viral RNA, co-factors (translated from NSP7 and NSP8), and helicase (translated from NSP13) (Hartenian et al., 2020). Additionally, there are several NSPs that exhibit protease and exoribonuclease activities for facilitating viral gene expression and disruption of the host cell signaling pathways, respectively (Perlman and Netland, 2009).

Meanwhile, accessory proteins of SARS-CoV-2 are considered as major virulence factors that counteract the host immune system, regulate cell death, and induce autophagy to provide a site for viral replication. For example, ORF3a can manipulate host responses, modulating autophagy, cell death, and immune response; while another accessory proteins such as ORF6, ORF7, and ORF8, can mitigate type I/III IFN responses or inflammatory responses (Ren et al., 2020; Xia et 
Table 1. Virological and immunological role of SARS-CoV-2-encoded proteins

\begin{tabular}{|c|c|c|c|c|}
\hline Viral protein & Virological function & Immunological function & Target pathway & Reference \\
\hline $\mathbf{N}$ & $\begin{array}{l}\text { Virion structure, } \\
\text { RNA packaging }\end{array}$ & $\begin{array}{l}\text { Inhibition of TRIM25-RIG-I and TBK1-IRF3 interaction } \\
\text { Disruption of TBK1, IRF3, and STAT1/2 activation } \\
\text { Suppression of MAVS oligomerization }\end{array}$ & $\begin{array}{l}\text { RLR activation } \\
\text { IFN production }\end{array}$ & $\begin{array}{l}\text { Chen et al. }(2020 \mathrm{a}) \\
\text { Lei et al. }(2020) \\
\text { Mu et al. }(2020) \\
\text { Oh and Shin (2021) }\end{array}$ \\
\hline $\mathbf{M}$ & Virion structure & $\begin{array}{l}\text { Inhibition of RIG-I-MAVS, MAVS-TBK1, and TRAF3-TBK1 interaction } \\
\text { Disruption of IRF3 activation } \\
\text { Suppression of MAVS oligomerization }\end{array}$ & $\begin{array}{l}\text { RLR activation } \\
\text { IFN production }\end{array}$ & $\begin{array}{l}\text { Lei et al. }(2020) \\
\text { Xia et al. }(2020) \\
\text { Zheng et al. }(2020) \\
\text { Fu et al. }(2021)\end{array}$ \\
\hline NSP1 & $\begin{array}{l}\text { Non-structural } \\
\text { protein }\end{array}$ & $\begin{array}{l}\text { Cleavage of host mRNAs } \\
\text { Inhibition of host protein translation and IFN response }\end{array}$ & $\begin{array}{l}\text { IFN activation } \\
\text { production }\end{array}$ & $\begin{array}{l}\text { Banerjee et al. (2020) } \\
\text { Schubert et al. }(2020) \\
\text { Thoms et al. }(2020) \\
\text { Vazquez et al. }(2021)\end{array}$ \\
\hline NSP3 & PLpro & $\begin{array}{l}\text { Inhibition of MDA5 activation } \\
\text { Proteolysis of IRF3 } \\
\text { de-ISGylation and de-ubiquitination of ISG15 }\end{array}$ & $\begin{array}{l}\text { RLR activation } \\
\text { IFN production }\end{array}$ & $\begin{array}{l}\text { Klemm et al. (2020) } \\
\text { Shin et al. (2020) } \\
\text { Liu et al. (2021) } \\
\text { Moustaqil et al. (2021) }\end{array}$ \\
\hline NSP5 & 3CLpro & $\begin{array}{l}\text { Cleavage of NLRP12 and TAB1 } \\
\text { Disruption of IRF3 nuclear translocation }\end{array}$ & $\begin{array}{l}\text { IFN activation \& } \\
\text { production } \\
\text { Inflammation }\end{array}$ & Moustaqil et al. (2021) \\
\hline NSP6 & DMV formation & $\begin{array}{l}\text { Restricts host autophagosome expansion } \\
\text { Disruption of IRF3 and STAT1/2 activation }\end{array}$ & $\begin{array}{l}\text { IFN activation \& } \\
\text { production }\end{array}$ & Xia et al. (2020) \\
\hline NSP7/8 & RNA primase & $\begin{array}{l}\text { Suppression of K63-ubiquitination of MDA5 } \\
\text { Attenuation of host translational system }\end{array}$ & $\begin{array}{l}\text { RLR activation } \\
\text { IFN activation \& } \\
\text { production }\end{array}$ & $\begin{array}{l}\text { Banerjee et al. }(2020) \\
\text { Yang et al. }(2020)\end{array}$ \\
\hline NSP12 & $\operatorname{RdRp}$ & Disruption of IRF3 and STAT1 activation & $\begin{array}{l}\text { IFN production } \\
\text { signaling }\end{array}$ & Wang et al. (2021b) \\
\hline NSP13 & NTPase, Helicase & $\begin{array}{l}\text { Disruption of TBK1, IRF3, and STAT1/2 activation } \\
\text { Interference with USP13-mediated ubiquitination }\end{array}$ & $\begin{array}{l}\text { IFN production } \\
\& \text { signaling }\end{array}$ & $\begin{array}{l}\text { Xia et al. }(2020) \\
\text { Yuen et al. }(2020) \\
\text { Guo et al. }(2021) \\
\text { Vazquez et al. }(2021)\end{array}$ \\
\hline NSP14 & $\begin{array}{l}\text { ExoN, } \\
\text { Guanine-N7-MTase }\end{array}$ & $\begin{array}{l}\text { Prevention of RIG-I mediated signaling and IRF3 activation } \\
\text { Suppression of host translational system } \\
\text { Downregulation of ISG15 and IFNAR1 expression }\end{array}$ & $\begin{array}{l}\text { IFN activation \& } \\
\text { production } \\
\text { ISG expression }\end{array}$ & $\begin{array}{l}\text { Yuen et al. }(2020) \\
\text { Hayn et al. }(2021) \\
\text { Hsu et al. }(2021)\end{array}$ \\
\hline NSP15 & NendoU & Disruption of IRF3 activation & IFN activation & Yuen et al. (2020) \\
\hline NSP16 & $\begin{array}{l}2^{\prime} \text {-O-MTase, } \\
\text { Viral mRNA capping }\end{array}$ & $\begin{array}{l}\text { Prevention of MDA5- mediated signaling and IRF3 activation } \\
\text { Inhibition of mRNA splicing system } \\
\text { Downregulation of ISG expression }\end{array}$ & $\begin{array}{l}\text { IFN production } \\
\text { ISG expression }\end{array}$ & Banerjee et al. (2020) \\
\hline ORF3b & Accessory protein & Disruption of IRF3 activation & $\begin{array}{l}\text { IFN activation \& } \\
\text { production }\end{array}$ & Konno et al. (2020) \\
\hline ORF6 & Accessory protein & $\begin{array}{l}\text { Disruption of IRF } 3 / 7 \text { and STAT } 1 / 2 \\
\text { Interruption of nucleocytoplasmic transport system } \\
\text { Downregulation of IFNAR } 1 \text { expression }\end{array}$ & $\begin{array}{l}\text { IFN production } \\
\& \text { signaling } \\
\text { ISG expression }\end{array}$ & $\begin{array}{l}\text { Li et al. }(2020 \mathrm{~b}) \\
\text { Miorin } \text { et a. }(2020) \\
\text { Addetia et al. }(2021) \\
\text { Kato } \text { et al. }(2021)\end{array}$ \\
\hline ORF7a & Accessory protein & Inhibition of STAT1 phosphorylation & IFN signaling & Xia et al. (2020) \\
\hline ORF7b & Accessory protein & Disruption of STAT1 and STAT2 phosphorylation & IFN signaling & Xia et al. (2020) \\
\hline ORF8 & Accessory protein & $\begin{array}{l}\text { Disruption of IRF3 activation } \\
\text { Induction of cytokine storm }\end{array}$ & $\begin{array}{l}\text { IFN activation } \\
\text { Inflammation }\end{array}$ & $\begin{array}{l}\text { Li et al. }(2020 \mathrm{~b}) \\
\text { Lin et al. }(2021) \\
\text { Rashid et al. }(2021)\end{array}$ \\
\hline ORF9b & Accessory protein & Inhibition of MAVS/TRAF3/TRAF6 complex & $\begin{array}{l}\text { RLR activation } \\
\text { IFN activation } \\
\text { production }\end{array}$ & $\begin{array}{l}\text { Han et al. }(2021) \\
\text { Wu et al. }(2021)\end{array}$ \\
\hline
\end{tabular}

Pro, protease; DMV, double membrane vesicle; RLRs, RIG-I-like receptors; RdRp, RNA-dependent RNA polymerase; NTPase, Nucleoside-triphosphatase; ExoN, Exonuclease; MTase, Methyltransferase; NendoU, Nidoviral uridylate-specific endoribonuclease; IFN, interferon;

al., 2020; Miao et al., 2021).

In this review, we highlight the recently described role of antiviral interferon (IFN) pathways in limiting SARS-CoV-2 infection, as well as the molecular mechanisms by which the virus evades the IFN response. We will also comprehensively review recent work showing the modulation of the IFN pathway by SARS-CoV-2-encoded proteins (Table 1). A detailed understanding of the mechanisms by which SARS$\mathrm{CoV}-2$ suppresses host antiviral pathways may provide novel avenues for the development of antiviral drugs against SARSCoV-2.

\section{SARS-CoV-2 Recognition by RIG-I-like Receptors (RLRs) and Toll-like receptors (TLRs)}

To defend against SARS-CoV-2, innate immune responses are activated to produce antiviral factors, especially type I 
and III IFN, which are rapidly produced by immune or epithelial cells. For IFN production, pattern recognition receptor (PRR) signaling pathways are activated by conserved pathogen-associated molecular patterns (PAMPs) that initiate a cascade of innate immune responses (Fitzgerald and Kagan, 2020). Among PRR signaling pathways, RLR and TLR pathways are highly important for the recognition of viral components and further lead to antiviral immune response, as depicted in Fig. 1.

RLRs, such as retinoic acid inducible gene-I (RIG-I) and melanoma differentiation-associated gene 5 (MDA5), are typically expressed in most cell types and sense viral RNA to elicit IFN response (Rehwinkel and Gack, 2020). While RIG-I recognizes viral RNA with $5^{\prime}$-triphosphate or short dsRNA sequences, MDA5 senses long dsRNA with no end specificity (Reikine et al., 2014). Once the ATP-dependent DExD/H box RNA helicase domain of RLR proteins binds to viral RNA, these sensors interact with the $\mathrm{N}$-terminal caspase activation and recruitment domains (CARDs) of mitochondrial antiviral signaling protein (MAVS) and coordinates downstream signaling pathways by recruiting ubiquitin ligases, such as TNF receptor associated factors (TRAFs), TANK-binding kinase 1 (TBK1), IKK- $\varepsilon$ and IKK- $\alpha / \beta / \gamma$ complex. As a consequence, several transcriptional factors such as IRF3, IRF7 and NF- $\kappa$ B get phosphorylated and translocated to nucleus, thereby triggering the expression of IFNs or pro- inflammatory cytokines. IFNs are produced and released in both autocrine and paracrine ways and promote hundreds of interferon stimulated genes (ISGs), which are capable of interfering with every steps of viral replication (Schneider et al., 2014).

Multiple studies reported that the RLR pathway is activated during SARS-CoV-2 infection for anti-viral responses in vitro (Kouwaki et al., 2021; Sampaio et al., 2021; Thorne et al., 2021; Wu et al., 2021; Yamada et al., 2021; Yin et al., 2021). For example, Thorne et al. (2021) have reported that RNA sensing of SARS-CoV-2 in lung epithelium can contribute to robust macrophage activation, cytokine production and tissue inflammation. Meanwhile, both Yin and Sampaio et al. reported that SARS-CoV-2 is sensed by MDA5, NOD1, and LGP2, but not RIG-I and MAVS; while IRF3, IRF5, and NF-kB/p65 act as the major mediators of IFN response induction (Sampaio et al., 2021; Yin et al., 2021).

In addition to RLRs, TLRs are also considered critical components of host defense against SARS-CoV-2. TLRs comprise of ten members in human and are expressed in the cell membrane or endosomes. Each TLR recognizes different PAMPs, including lipopolysaccharide (recognized by TLR4), peptidoglycan (recognized by TLR2), flagellin (recognized by TLR5), and CpG DNA (recognized by TLR9). The TLR pathway involves activation of downstream adaptor molecules and divides into MyD88- and TRIF-dependent pathways (Fitz-

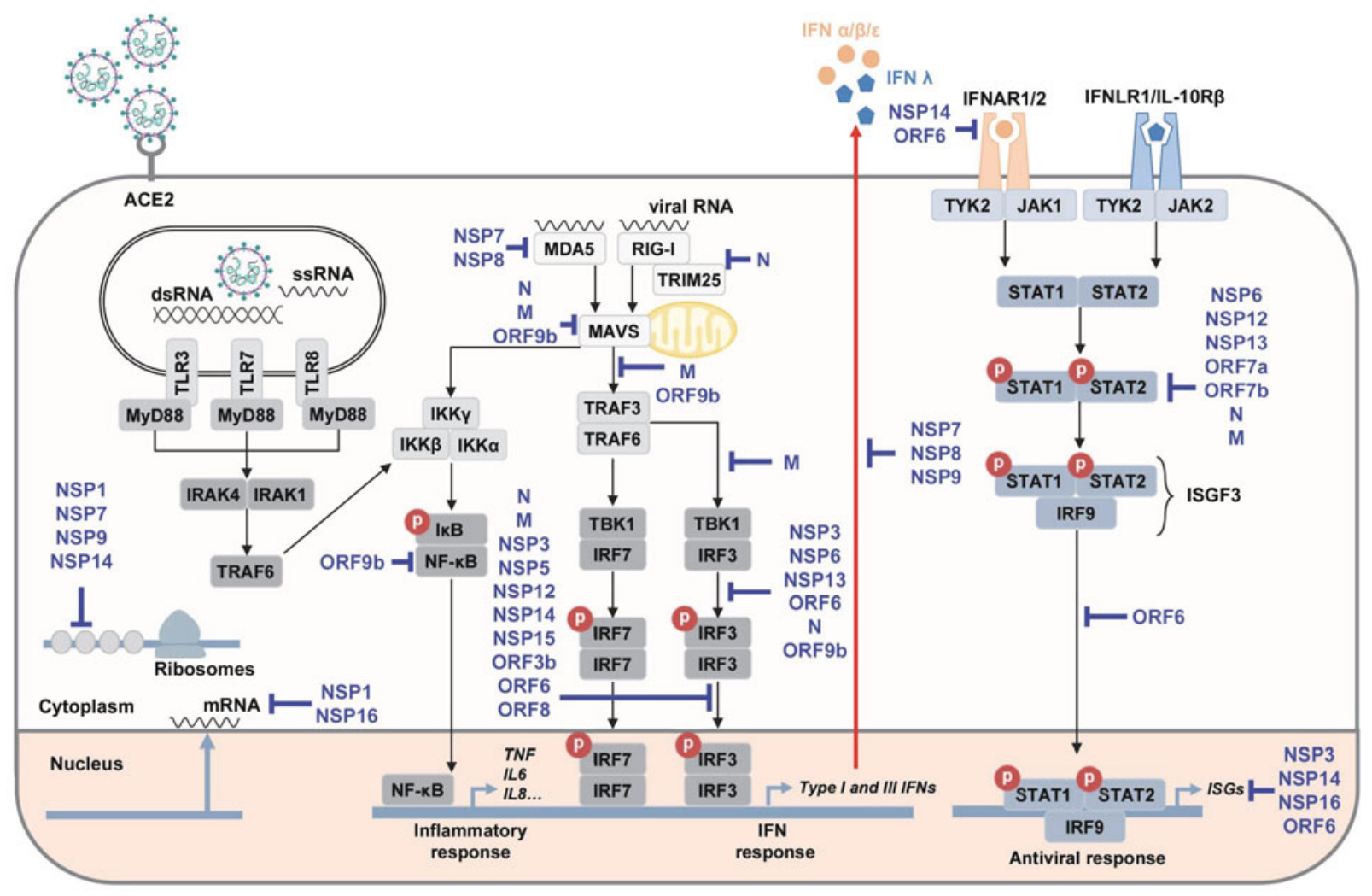

Fig. 1. SARS-CoV-2 proteins antagonize multiple steps of antiviral response. The multiple host factors in the antiviral signaling cascade are targeted by SARS$\mathrm{CoV}-2$ proteins. During SARS-CoV-2 infection, viral recognition by host innate immune sensors such as RLRs and TLRs triggers an antiviral signaling cascade to elicit the production of type I or type III IFN as well as proinflammatory cytokines. To combat against host defense mechanisms, SARS-CoV-2 have evolved multiple strategies to avoid host recognition by interfering with the function of antiviral proteins using various viral proteins. Indicated in blue are sites at which the SARS-CoV-2 interferes with host innate immune sensing pathways, as explained in the text. 
gerald and Kagan, 2020). All TLRs except TLR3 use MyD88dependent pathway, whereas TLR3 as well as TLR4 induces TRIF-dependent pathway, which activates TBK1 and IKK- $\varepsilon$ to phosphorylate IRF3 or IRF7 to propagate IFN responses. Among TLRs, TLR3 and TLR7/TLR8 heterodimer can recognize dsRNA or ssRNA from viruses, respectively and therefore, is likely to be emphasized in the restriction of SARSCoV-2.

So far, several TLRs have been shown to be involved in pathogenesis of SARS-CoV-2 infection. As an example, Zheng et al. (2021) has indicated that TLR2 is important for SARS$\mathrm{CoV}-2$ recognition. E protein of SARS-CoV-2 is able to stimulate TLR2/MyD88 signaling pathway and blocking TLR2 signaling in vivo resulted in protection against SARS-CoV-2 infection. Additionally, TLR4 was shown to directly interact with the S protein of SARS-CoV-2 (Zhao et al., 2021a) and the $\mathrm{S} 1$ domain of the $\mathrm{S}$ protein was shown to activate NF- $\mathrm{\kappa B}$ pathway, which is dependent on TLR4 in macrophages (Shirato and Kizaki, 2021). Given that TLR3 is essential for protection against SARS-CoV by elevating the production of pro-inflammatory cytokines and IFNs in mice (Totura et al., 2015), it is highly likely that TLR3 may contribute to protection against SARS-CoV-2 infection (Iwasaki and Yang, 2020). In fact, SARS$\mathrm{CoV}-2$ infection increased TLR3-mediated NF- $\kappa \mathrm{B}$ signaling pathway and TLR3 antagonist, famotidine, which is an approved drug for peptic ulcers and gastroesophageal reflux disease, mitigates SARS-CoV-2-induced inflammation (Mukherjee et al., 2021). Lastly, TLR7/8 was also demonstrated in SARS-CoV-2 ssRNA recognition, subsequently leading to dendritic cell activation and $\mathrm{T}$ cell inflammatory response (Salvi et al., 2021).

\section{The Role of IFNs in SARS-CoV-2 Infection}

IFNs are critical components of host defense against viruses. IFNs are secreted from virus-infected cells, bind to IFN receptors on bystander cells in an autocrine or paracrine manner and strongly induce ISGs for an antiviral response (Park and Iwasaki, 2020). Type I (IFN- $\alpha$, IFN- $\beta$, and IFN- $\varepsilon$ ) and III (IFN- $\lambda$ ) IFNs can activate receptor associated tyrosine kinase TYK2 and JAK1, subsequently phosphorylating signal transducer and activator of transcription proteins (STATs), such as STAT1 and STAT2. Together with IRF9, STAT1, STAT2 form IFN-stimulated gene factor 3 (ISGF3) complex, which translocate to the nucleus upon phosphorylation and activate the interferon stimulatory response element (ISRE) promoter to express ISGs. So far, there are hundreds of ISGs discovered, and many ISGs exert antiviral functions by inhibiting viral gene expression and eliminating viral components.

The role of type I and III IFN response in COVID-19 has been examined by several groups. First, multiple groups have reported that SARS-CoV-2 infection is sensitive to IFN pretreatment or production in vitro (Miorin et al., 2020; Hayn et al., 2021; Yin et al., 2021). Moreover, the dysregulated type I and III IFN response strongly contributes to the pathogenesis of COVID-19. Zhang et al. (2020) have revealed that that patients with genetic mutations in TLR pathways, including TLR3 and IRF7, show severe symptoms including death, as these mutations hamper type I IFN responses. The same group also reported that autoantibodies against type I IFNs, including IFN- $\omega$ and multiple subsets of IFN- $\alpha$, were produced in $10 \%$ of patients with critical symptoms, in contrast to $0.3 \%$ of patients with mild symptoms. Further, the patients who produce autoantibodies also show a lower level of IFN- $\alpha$ production, and the autoantibodies against IFNs can neutralize type I IFN response in such patients (Bastard et al., 2020). While it is certain that IFNs play important role in anti-viral responses, they have also been reported to enhance the expression of ACE2, thereby possibly contributing to the higher infectivity of SARS-CoV-2 (Busnadiego et al., 2020; Ziegler et al., 2020). Despite the contradictory roles of IFNs in SARS-CoV-2 infection, there is no doubt that interventions with IFN responses can play a significant role in restricting SARS-CoV-2 infection and replication.

Multiple groups have reported that a distinct immune phenotype is observed in COVID-19 patients, wherein COVID19 patients with severe symptoms exhibit defective innate and adaptive immune responses, including hypercytokinemia, T cell loss, dysfunctional NK cells, and shortened memory $\mathrm{T}$ cell responses (Yang et al., 2021). In particular, significant imbalances in the secretion of cytokines and chemokines have been detected in patients with severe COVID-19 as compared to that in patients with mild symptoms. Although impaired production of type I IFNs have been suggested to be responsible for detrimental outcomes of COVID-19 (Acharya et al., 2020; Blanco-Melo et al., 2020; Hadjadj et al., 2020), robust IFN responses were detected in severe COVID-19 patients along with elevated production of pro-inflammatory cytokines and chemokines, including TNF- $\alpha$, IL- 6 , and IL- $1 \beta$ (Lee et al., 2020). Given that cytokine storm can be worsened by delayed IFN production, the use of IFN in clinical settings should be carefully evaluated; and further studies on the detailed mechanisms of innate immune sensing and the role of IFNs in SARS-CoV-2 infected cells are essential.

\section{IFN Antagonisms by SARS-CoV-2 Structural Proteins}

\section{Nucleocapsid (N) protein}

$\mathrm{N}$ protein encoded by coronaviruses has a function in the packaging of RNA into structural proteins and is involved in viral replication and release. Similar to SARS-CoV (Lu et al., 2011; Hu et al., 2017), multiple groups, including ours, have shown that SARS-CoV-2 N protein can interfere with RLR pathways and thus antagonize IFN production and signaling (Chen et al., 2020a; Lei et al., 2020; Mu et al., 2020; Oh and Shin, 2021). In detail, SARS-CoV-2 N inhibits TRIM25RIG-I interaction, TBK1-IRF3 interaction and represses the phosphorylation of TBK1 and IRF3, and nuclear translocation of IRF3 induced by poly I:C or RNA viruses (Chen et al., 2020a; Gori Savellini et al., 2021; Oh and Shin, 2021). Another proposed mechanism of IFN antagonism by N protein targets K63-linked polyubiquitination and oligomerization of MAVS (Wang et al., 2021a; Zotta et al., 2021). The dimerization domain of the $\mathrm{N}$ protein, which forms small droplets to isolate viral RNA, inhibits MAVS oligomerization and further leads to delayed type I IFN response both in vivo and in vitro. Interestingly, a dual role of the $\mathrm{N}$ pro- 
tein in the regulation of IFN response was recently proposed (Zhao et al., 2021b). A low dose of $\mathrm{N}$ protein has the function of IFN antagonism; however, a high dose of $\mathrm{N}$ protein induces robust type I IFN signaling and inflammation. Along with interfering RLR pathways, the inhibition of JAK/STAT pathway by $\mathrm{N}$ protein also has been reported (Mu et al., 2020). $\mathrm{N}$ proteins can inhibit phosphorylation of STAT1 and 2 stimulated by IFN- $\beta$ treatment by directly interacting with STAT1 and STAT2. Given that emerging variants of SARS$\mathrm{CoV}-2$ contain genetic mutations in $\mathrm{N}$ proteins (Toyoshima et al., 2020), it will be important to characterize the effect of $\mathrm{N}$ mutations on IFN responses.

\section{Membrane (M) protein}

$\mathrm{M}$ protein is the most abundant structural protein expressed in the envelope of coronaviruses. SARS-CoV-2 M protein can localize in the endoplasmic reticulum (ER) and Golgi and possess the first transmembrane domain (TM1) at the N-terminus to localize in the Golgi (Zheng et al., 2020). SARSCoV-2 M protein suppresses type I/III IFN response induced by poly I:C or RNA virus infection and interacts with RIG-I, MDA5, MAVS, TRAF3, and TBK1. M protein inhibits RIGI-MAVS, MAVS-TBK1, TRAF3-TBK1 interaction, and finally, downregulates the phosphorylation and nuclear translocation of IRF3. Meanwhile, TM1-lacking M protein no longer interacts with RIG-I, but still interacts with MAVS and TBK1 (Lei et al., 2020; Xia et al., 2020; Zheng et al., 2020). More recently, one group further reported IFN-antagonizing properties of M protein by inhibition of MAVS aggregation (Fu et al., 2021). Taken together, M protein serves as IFN antagonist via interaction with RLR molecules and inhibits the oligomerization of MAVS, which in turn suppresses the phosphorylation and nuclear translocation of IRF3 and blocks IFN signaling.

\section{IFN Antagonism by SARS-CoV-2 NSPs}

\section{NSP1}

NSP1 is an IFN antagonist (Lei et al., 2020; Xia et al., 2020), which hampers IFN responses, cleaves host mRNAs and suppress protein translation (Banerjee et al., 2020; Schubert et al., 2020; Thoms et al., 2020; Vazquez et al., 2021). In detail, NSP1 downregulates mRNA translation in the host cells by interacting with the $18 \mathrm{~S}$ ribosomal RNA and $40 \mathrm{~S}$ ribosomal subunit in vivo and in vitro. In addition, blocking the host translation system subsequently reduces RIG-I and ISG15 protein expression, resulting in decreased ISRE promoter activity (Thoms et al., 2020). Given that NSP1 interacts with nuclear export receptor NXF1 and shutdowns poly (A) RNA export system, NSP1 offers potential uncovered strategies for interrupting the host translational system to inhibit the IFN response (Zhang et al., 2021b).

\section{NSP3}

NSP3 is a papain-like protease, which contains multiple domains necessary for viral replication, gene transcription, and protein translation. As an immune evasion strategy, NSP3 physically interacts with IRF3 and inhibits IRF3 phospho- rylation, dimerization, and translocation (Klemm et al., 2020; Shin et al., 2020). In addition to targeting IRF3 activation, NSP3 induces de-ISGylation and de-ubiquitinylation of ISG15, which subsequently attenuates ISG15-dependent activation of MDA5 (Shin et al., 2020; Liu et al., 2021). Similar to other viral proteins with protease activity, which induce cleavage or degradation of host proteins associated with antiviral signaling to evade the host immune response, the papain-like protease activity of NSP3 also shows the proteolysis of IRF3 (Moustaqil et al., 2021). Considering that the NSP3 acts to inhibit host innate immunity via protease activity, it will be essential to develop protease inhibitors particularly targeting NSP3.

\section{NSP5}

Similar to NSP3, NSP5 is a protease, which contributes to viral replication via processing viral polyproteins through 3C-like protease (3CLpro) activity. NSP5 was detected to cleave nucleotide-binding leucine-rich domain containing receptors, NLRP12 and TAB1, which are involved in the inflammatory response (Moustaqil et al., 2021). Along with the downregulation of inflammation, NSP5 also inhibits the nuclear translocation of IRF3 without affecting the phosphorylation of IRF3, resulting in decreased IFN- $\beta$ luciferase activity (Fung et al., 2021).

\section{NSP6}

Previous studies have revealed that SARS-CoV NSP6 can generate ER-derived autophagosomes by forming host double-membrane vesicles (DMV) and facilitating viral replication and translation (Cottam et al., 2011). In regard to this, it will be interesting to see if SARS-CoV-2 NSP6 can also sequester the autophagy pathway to inhibit the formation of the autolysosome. Meanwhile, NSP6 interferes with the host immune response by interacting with TBK1 to inhibit the phosphorylation and nuclear translocation of IRF3 (Xia et al., 2020). In particular, NSP6 inhibits not only the RLR signaling but also the JAK/STAT pathway. Overexpression of NSP6 in cells show lower activation and cytoplasmic localization of STAT1 and STAT2 during IFN- $\alpha$ treatment. Furthermore, NSP6 has also been shown to significantly inhibit STAT1 and STAT2 as compared to that of other coronaviruses such as SARS-CoV, and MERS-CoV (Xia et al., 2020).

\section{NSP7/8}

NSP7 forms heterodimers with NSP8 and plays a role in facilitating the RNA-dependent RNA polymerase encoded by NSP12 (Kasuga et al., 2021). NSP8 directly interacts with MDA5 via the CARD domain and inhibits MDA5-mediated ISRE luciferase activity by suppressing K63-linked poly-ubiquitination of MDA5 (Yang et al., 2020). Intriguingly, NSP7 can interfere with NSP1; which is associated with host translational machinery. Furthermore, NSP8 and NSP9 bind to the signal recognition particle complex, 7SL RNA, to inhibit the host protein trafficking system from secreting proteins (Banerjee et al., 2020). These findings imply that NSP8 and NSP9 can inhibit several cytokines or chemokines that are involved in the innate immune response, including type I/III IFN or proinflammatory responses. 


\section{NSP12}

NSP12 is an RNA-dependent RNA polymerase that requires the NSP7-NSP9 heterodimer as a processivity factor responsible for replication and methylation. NSP12 has been shown to dampen the RIG-I-, MDA5-, MAVS-, and IRF3-5D-mediated IFN- $\beta$ and ISRE luciferase activity (Wang et al., 2021b). In addition, NSP12 inhibits Sendai virus-induced phosphorylation of STAT1. On the other hand, Li et al. (2021) reported that NSP12 does not affect IRF3 phosphorylation, although it does prevent IRF3 nuclear translocation and inhibit the JAK/STAT pathway to evade the host antiviral response.

\section{NSP13}

NSP13 possesses NTPase and helicase activity and was first suggested as a potent IFN antagonist by Yuen et al. (2020). The mechanisms of IFN suppression by NSP13 have been reported by several groups. In particular, NSP13 has been shown to inhibit TBK1 and IRF3 phosphorylation by interacting with TBK1 and interfere with the JAK/STAT pathway (Xia et al., 2020; Vazquez et al., 2021). Furthermore, Guo et al. (2021) have reported that NSP13 also interferes with the host deubiquitinase USP13 to stabilize itself and attenuate host IFN responses.

\section{NSP14}

NSP14 possess exonuclease and methyltransferase activity for viral gene modification. In addition to being a viral replisome, NSP14 is another inhibitor of IRF3 nuclear translocation that disrupts RIG-I-mediated IFN- $\beta$ production but not IFN- $\beta$-induced ISRE promoter activities (Yuen et al., 2020). NSP14 interferes with the host translational system as well as the RLR pathway to manipulate the host immune system, similar to NSP1. Also, NSP14 associates with NSP10 to block the host translational machinery to attenuate ISG expression in NSP14-expressing VeroE6 cells. Thus, the catalytic activity of NSP14 contributes to the inhibition of host protein translation (Hsu et al., 2021). Together with the manipulation of the translational system, NSP14 can repress IFNAR1 protein expression by lysosomal degradation (Hayn et al., 2021).

\section{NSP15}

NSP15 is a nidoviral uridylate-specific endoribonuclease that processes viral RNA in its mature form. Yuen et al. (2020) have demonstrated NSP15's ability to antagonize IFN production by inhibition of IRF3 translocation, but the specific mechanisms by which NSP15 mediates IFN inhibition remain to be fully examined.

\section{NSP16}

Similar to NSP13, 14, and 15, NSP16 is an RNA-modifying enzyme that methylates the $2^{\prime}-\mathrm{O}$ position to modify the viral genome in association with NSP10. NSP16 is involved in interrupting mRNA splicing mechanisms. NSP16 has been shown to bind with $\mathrm{U} 1$ and $\mathrm{U} 2$, which are components of the spliceosome, to suppress host mRNA splicing and thereby result in decreased ISG expression (Banerjee et al., 2020).

\section{IFN Antagonisms by SARS-CoV-2 Accessory Proteins}

\section{ORF3}

ORF3a was first identified as a pro-apoptotic factor (Ren et al., 2020), whereas ORF3b robustly reduces IFN- $\beta$ promoter activity to inhibit type I IFN response by inhibiting IRF3 translocation during Sendai virus infection (Lei et al., 2020) and the antiviral effect is influenced by C-terminal length of the protein (Konno et al., 2020). Moreover, the patients infected the SARS-CoV-2 strains with ORF3b variants developed more severe symptoms as compared to the other patients infected with the originally identified SARS-CoV-2. These findings thus implicate the IFN-suppressive activity of ORF3b as an important virulence factor in the pathogenesis of SARS-CoV-2 (Konno et al., 2020).

\section{ORF6}

SARS-CoV-2 ORF6 is known to antagonize IFN responses (Yuen et al., 2020; Kimura et al., 2021), whereas SARS-CoV ORF6 was shown to disrupt the nuclear translocation of IRF3 and STAT1 (Frieman et al., 2007; Kopecky-Bromberg et al., 2007). Consistent with this, SARS-CoV-2 ORF6 can also antagonize IFN responses by disrupting the nucleocytoplasmic transport system (Miorin et al., 2020; Addetia et al., 2021; Kato et al., 2021). Specifically, Miorin et al. (2020) have reported that SARS-CoV-2-infected VeroE6 cells show the localization of STAT1 and STAT2 in the cytoplasm, not in the nucleus, as a result of ORF6-NUP98-RAE1 complex formation and especially the ORF6-NUP98 interaction. Similarly, the association of ORF6, NUP98, and RAE1 has been shown to disrupt the nuclear translocation of IRF3 (Kimura et al., 2021) and the association strength of the ORF6-NUP98-RAE1 complex is stronger than that of SARS-CoV-1 ORF6 (Addetia et al., 2021). Furthermore, Kato et al. (2021) have reported that SARS-CoV-2 ORF6 induces mislocalization of RAE1. Together with the interference from NUP98-RAE1 complex, ORF6 also binds with karyopherin $\alpha 2$, which is an important factor for the nuclear localization of IRF3, IRF7, and STAT1, to block the nuclear translocation of IRF3 (Xia et al., 2020). Furthermore, ORF6 has the potential to disrupt the JAK/STAT pathway as ORF6 represses the expression of IFNAR1 (Hayn et al., 2021). Taken together, the studies suggest that ORF6 exploits IFN pathways via inhibition of IRF3 and STAT nuclear translocation.

\section{ORF7}

ORF7 is divided into ORF7a and ORF7b depending on translation initiation sites; both ORFs can abrogate the JAK/STAT pathway to evade the host immune response (Xia et al., 2020). Both ORF7a and ORF7b have been shown to strongly inhibit ISRE luciferase activity induced by recombinant IFN- $\alpha$ treatment as compared to unstimulated conditions. While ORF7a has been shown to inhibit STAT1 phosphorylation, ORF7b has been demonstrated to inhibit both STAT1 and STAT2 (Cao et al., 2021). 


\section{ORF8}

SARS-CoV-2 ORF8 is an accessory protein that encodes ER import sequences to facilitate ER localization and shows very low sequence identity as compared to that of SARS-CoV. The function of IFN antagonism by ORF8 was first proposed by Li et al. (2020b) suggesting that ORF8 attenuates the promoter activities of IFN- $\beta$, ISRE, and NF- $\mathrm{kB}$ and the expression of ISGs. Furthermore, ORF8 inhibits the nuclear translocation of IRF3 to suppress the IFN- $\beta$ gene expression (Rashid et al., 2021). Another group, Zhang et al. (2021a), reported that ORF8 is capable of modulating adaptive immune response by autophagy-mediated MHC-I degradation and downregulation, however, the detailed mechanisms by which ORF8 hijacks IFN responses have not been fully elucidated. Given that ORF8 of SARS-CoV-2 has been proposed to play a role in ER stress response, unfolded protein response (UPR), and autophagy process by interacting host proteins involved in these pathways, further studies on the immunological effect of ORF8 will be necessary (Gordon et al., 2020).

\section{ORF9b}

ORF9b is expressed rapidly during SARS-CoV-2 infection and strongly co-localizes with mitochondria as well as with ER and Golgi in ORF9b-transfected HeLa cells (Han et al., 2021). In SARS-CoV, ORF9b attenuates type I IFN response by targeting MAVS/TRAF3/TRAF6 complex that are expressed on the mitochondria (Shi et al., 2014). Similarly, SARS-CoV-2-ORF9b also targets the mitochondrial protein, TOM70, to repress the antiviral signaling (Jiang et al., 2020). Wu et al. (2021) reported that ORF9b attenuates both type I IFN and inflammatory response by targeting RIG-I-MAVS signaling pathways. The proposed mechanisms of IFN antagonism by ORF9b involve the suppression of NF- $\mathrm{KB}$ activation and K63-linked polyubiquitination of NEMO. Overexpression of ORF9b suppresses DNA sensing pathways by interacting with RIG-I, MDA5, MAVS, TBK1, STING, and TRIF (Han et al., 2021). As ORF9b is expressed rapidly during SARS-CoV-2 infection, IFN antagonism of ORF9b is attributed to be one of the causes of delayed immune responses in COVID-19 patients.

\section{Concluding Remarks}

Viral evasion of host innate immunity is necessary for successful virus replication and thus, it is essential to characterize mechanical insights into how SARS-CoV-2 antagonizes antiviral interferon response. What makes SARS-CoV-2 highly pathogenic and deadly in certain population? It is very likely that SARS-CoV-2's ability to circumvent and escape antiviral immunity may contribute to failure of appropriate and timely activation of host antiviral defense mechanisms and instead lead to successful viral replication. Extensive studies in the past two years have provided insights into how each viral factors of SARS-CoV-2 may avoid, subvert or interfere with antiviral innate immune signaling pathways. However, many questions still remained unanswered, including which genes are required for transmission to a new host despite the robust immune response; Are there a functional redundancy for multiple virulent factors? Additionally, are there tissueor cell type specificity of how these virulence factors may work? Are there functions of viral factors modulated by its genetic context and is there any evidence for cooperativity? To date, although we have accumulating evidences to show each viral factor's function and structure by ectopic expression, further studies involving the construction of virus mutants and in vivo functional analysis of viral factors in terms of transmission and pathogenesis are yet to begin. Functional analysis of viral factors in the context of virus infection in vitro and in vivo should be further investigated to understand better about the complex interaction between viruses and host.

Despite massive efforts to control the COVID-19 pandemic, we are still facing multiple challenges concerning the longterm efficacy of vaccines as well as their effectiveness against present and future variants. Therefore, it is essential to have a better understanding of the interplay between the immune system and the virus in addition to the cells or molecules that drive disease pathology to develop proper therapeutic interventions. As millions of people will be suffering from long-term effects of COVID-19 even after the pandemic is controlled by vaccination, we must continue to improve our understanding of the underlying pathogenesis of SARS-CoV-2 to better equip ourselves against such threats in the future.

\section{Acknowledgements}

This research was funded by the Basic Science Research Program of the National Research Foundation of Korea (NRF) by the Ministry of Science, ICT \& Future Planning (NRF2019R1A2C1005961), and Korea Health Technology R\&D Project through the Korea Health Industry Development Institute (KHIDI), funded by the Ministry of Health \& Welfare, Republic of Korea (HI21C1252).

\section{Conflict of Interest}

The authors state no conflicts of interest.

\section{References}

Acharya, D., Liu, G., and Gack, M.U. 2020. Dysregulation of type I interferon responses in COVID-19. Nat. Rev. Immunol. 20, 397-398.

Addetia, A., Lieberman, N.A.P., Phung, Q., Hsiang, T.Y., Xie, H., Roychoudhury, P., Shrestha, L., Loprieno, M.A., Huang, M.L., Gale, M.Jr., et al. 2021. SARS-CoV-2 ORF6 disrupts bidirectional nucleocytoplasmic transport through interactions with Rael and Nup98. mBio 12, e00065-21.

Banerjee, A.K., Blanco, M.R., Bruce, E.A., Honson, D.D., Chen, L.M., Chow, A., Bhat, P., Ollikainen, N., Quinodoz, S.A., Loney, C., et al. 2020. SARS-CoV-2 disrupts splicing, translation, and protein trafficking to suppress host defenses. Cell 183, 1325-1339.

Bastard, P., Rosen, L.B., Zhang, Q., Michailidis, E., Hoffmann, H.H., Zhang, Y., Dorgham, K., Philippot, Q., Rosain, J., Béziat, V., et al. 2020. Autoantibodies against type I IFNs in patients with lifethreatening COVID-19. Science 370, eabd4585.

Blanco-Melo, D., Nilsson-Payant, B.E., Liu, W.C., Uhl, S., Hoagland, 
D., Møller, R., Jordan, T.X., Oishi, K., Panis, M., Sachs, D., et al. 2020. Imbalanced host response to SARS-CoV-2 drives development of COVID-19. Cell 181, 1036-1045.

Busnadiego, I., Fernbach, S., Pohl, M.O., Karakus, U., Huber, M., Trkola, A., Stertz, S., and Hale, B.G. 2020. Antiviral activity of type I, II, and III interferons counterbalances ACE2 inducibility and restricts SARS-CoV-2. $m$ Bio 11, e01928-20.

Cao, Z., Xia, H., Rajsbaum, R., Xia, X., Wang, H., and Shi, P.Y. 2021. Ubiquitination of SARS-CoV-2 ORF7a promotes antagonism of interferon response. Cell. Mol. Immunol. 18, 746-748.

Chen, K., Xiao, F., Hu, D., Ge, W., Tian, M., Wang, W., Pan, P., Wu, K., and Wu, J. 2020a. SARS-CoV-2 nucleocapsid protein interacts with RIG-I and represses RIG-mediated IFN- $\beta$ production. Viruses 13, 47.

Chen, N., Zhou, M., Dong, X., Qu, J., Gong, F., Han, Y., Qiu, Y., Wang, J., Liu, Y., Wei, Y., et al. 2020b. Epidemiological and clinical characteristics of 99 cases of 2019 novel coronavirus pneumonia in Wuhan, China: a descriptive study. Lancet 395, 507513.

Cottam, E.M., Maier, H.J., Manifava, M., Vaux, L.C., Chandra-Schoenfelder, P., Gerner, W., Britton, P., Ktistakis, N.T., and Wileman, T. 2011. Coronavirus nsp6 proteins generate autophagosomes from the endoplasmic reticulum via an omegasome intermediate. Autophagy 7, 1335-1347.

Cui, J., Li, F., and Shi, Z.L. 2019. Origin and evolution of pathogenic coronaviruses. Nat. Rev. Microbiol. 17, 181-192.

Fitzgerald, K.A. and Kagan, J.C. 2020. Toll-like receptors and the control of immunity. Cell 180, 1044-1066.

Frieman, M., Yount, B., Heise, M., Kopecky-Bromberg, S.A., Palese, P., and Baric, R.S. 2007. Severe acute respiratory syndrome coronavirus ORF6 antagonizes STAT1 function by sequestering nuclear import factors on the rough endoplasmic reticulum/golgi membrane. J. Virol. 81, 9812-9824.

Fu, Y.Z., Wang, S.Y., Zheng, Z.Q., Huang, Y., Li, W.W., Xu, Z.S., and Wang, Y.Y. 2021. SARS-CoV-2 membrane glycoprotein M antagonizes the MAVS-mediated innate antiviral response. Cell. Mol. Immunol. 18, 613-620.

Fung, S.Y., Siu, K.L., Lin, H., Yeung, M.L., and Jin, D.Y. 2021. SARSCoV-2 main protease suppresses type I interferon production by preventing nuclear translocation of phosphorylated IRF3. Int. J. Biol. Sci. 17, 1547-1554.

Gordon, D.E., Jang, G.M., Bouhaddou, M., Xu, J., Obernier, K., White, K.M., O’Meara, M.J., Rezelj, V.V., Guo, J.Z., Swaney, D.L., et al. 2020. A SARS-CoV-2 protein interaction map reveals targets for drug repurposing. Nature 583, 459-468.

Gori Savellini, G., Anichini, G., Gandolfo, C., and Cusi, M.G. 2021. SARS-CoV-2 N protein targets TRIM25-mediated RIG-I activation to suppress innate immunity. Viruses 13, 1439.

Guo, G., Gao, M., Gao, X., Zhu, B., Huang, J., Luo, K, Zhang, Y., Sun, J., Deng, M., and Lou, Z. 2021. SARS-CoV-2 non-structural protein 13 (nsp13) hijacks host deubiquitinase USP13 and counteracts host antiviral immune response. Signal Transduct. Target. Ther. 6, 119.

Hadjadj, J., Yatim, N., Barnabei, L., Corneau, A., Boussier, J., Smith, N., Péré, H., Charbit, B., Bondet, V., Chenevier-Gobeaux, C., et al. 2020. Impaired type I interferon activity and inflammatory responses in severe COVID-19 patients. Science 369, 718-724.

Han, L., Zhuang, M.W., Deng, J., Zheng, Y., Zhang, J., Nan, M.L., Zhang, X.J., Gao, C., and Wang, P.H. 2021. SARS-CoV-2 ORF9b antagonizes type I and III interferons by targeting multiple components of the RIG-I/MDA-5-MAVS, TLR3-TRIF, and cGASSTING signaling pathways. J. Med. Virol. 93, 5376-5389.

Hartenian, E., Nandakumar, D., Lari, A., Ly, M., Tucker, J.M., and Glaunsinger, B.A. 2020. The molecular virology of coronaviruses. J. Biol. Chem. 295, 12910-12934.

Hayn, M., Hirschenberger, M., Koepke, L., Nchioua, R., Straub, J.H., Klute, S., Hunszinger, V., Zech, F., Prelli Bozzo, C., Aftab, W., et al. 2021. Systematic functional analysis of SARS-CoV-2 pro- teins uncovers viral innate immune antagonists and remaining vulnerabilities. Cell Rep. 35, 109126.

Hoffmann, M., Kleine-Weber, H., Schroeder, S., Krüger, N., Herrler, T., Erichsen, S., Schiergens, T.S., Herrler, G., Wu, N.H., Nitsche, A., et al. 2020. SARS-CoV-2 cell entry depends on ACE2 and TMPRSS 2 and is blocked by a clinically proven protease inhibitor. Cell 181, 271-280.

Hsu, J.C.C., Laurent-Rolle, M., Pawlak, J.B., Wilen, C.B., and Cresswell, P. 2021. Translational shutdown and evasion of the innate immune response by SARS-CoV-2 NSP14 protein. Proc. Natl. Acad. Sci. USA 118, e2101161118.

Hu, Y., Li, W., Gao, T., Cui, Y., Jin, Y., Li, P., Ma, Q., Liu, X., and Cao, C. 2017. The severe acute respiratory syndrome coronavirus nucleocapsid inhibits type I interferon production by interfering with TRIM25-mediated RIG-I ubiquitination. J. Virol. 91, e02143-16.

Huang, C., Wang, Y., Li, X., Ren, L., Zhao, J., Hu, Y., Zhang, L., Fan, G., Xu, J., Gu, X., et al. 2020. Clinical features of patients infected with 2019 novel coronavirus in Wuhan, China. Lancet 395, 497506.

Iwasaki, A. and Yang, Y. 2020. The potential danger of suboptimal antibody responses in COVID-19. Nat. Rev. Immunol. 20, 339-341.

Jiang, H.W., Zhang, H.N., Meng, Q.F., Xie, J., Li, Y., Chen, H., Zheng, Y.X., Wang, X.N., Qi, H., Zhang, J., et al. 2020. SARS-CoV-2 ORF9b suppresses type I interferon responses by targeting TOM70. Cell. Mol. Immunol. 17, 998-1000.

Kasuga, Y., Zhu, B., Jang, K.J., and Yoo, J.S. 2021. Innate immune sensing of coronavirus and viral evasion strategies. Exp. Mol. Med. 53, 723-736.

Kato, K., Ikliptikawati, D.K., Kobayashi, A., Kondo, H., Lim, K., Hazawa, M., and Wong, R.W. 2021. Overexpression of SARS-CoV-2 protein ORF6 dislocates RAE1 and NUP98 from the nuclear pore complex. Biochem. Biophys. Res. Commun. 536, 59-66.

Kimura, I., Konno, Y., Uriu, K., Hopfensperger, K., Sauter, D., Nakagawa, S., and Sato, K. 2021. Sarbecovirus ORF6 proteins hamper induction of interferon signaling. Cell Rep. 34, 108916.

Klemm, T., Ebert, G., Calleja, D.J., Allison, C.C., Richardson, L.W., Bernardini, J.P., Lu, B.G.C., Kuchel, N.W., Grohmann, C., Shibata, Y., et al. 2020. Mechanism and inhibition of the papainlike protease, PLpro, of SARS-CoV-2. EMBO J. 39, e106275.

Konno, Y., Kimura, I., Uriu, K., Fukushi, M., Irie, T., Koyanagi, Y., Sauter, D., Gifford, R.J., USFQ-COVID19 Consortium, Nakagawa, S., et al. 2020. SARS-CoV-2 ORF3b is a potent interferon antagonist whose activity is increased by a naturally occurring elongation variant. Cell Rep. 32, 108185.

Kopecky-Bromberg, S.A., Martínez-Sobrido, L., Frieman, M., Baric, R.A., and Palese, P. 2007. Severe acute respiratory syndrome coronavirus open reading frame (ORF) $3 \mathrm{~b}$, ORF 6 , and nucleocapsid proteins function as interferon antagonists. J. Virol. 81, 548-557.

Kouwaki, T., Nishimura, T., Wang, G., and Oshiumi, H. 2021. RIGI-like receptor-mediated recognition of viral genomic RNA of severe acute respiratory syndrome coronavirus- 2 and viral escape from the host innate immune responses. Front. Immunol. 12, 700926 .

Krishnamoorthy, S., Swain, B., Verma, R.S., and Gunthe, S.S. 2020. SARS-CoV, MERS-CoV, and 2019-nCoV viruses: an overview of origin, evolution, and genetic variations. VirusDis. 31, 411-423.

Lee, J.S., Park, S., Jeong, H.W., Ahn, J.Y., Choi, S.J., Lee, H., Choi, B., Nam, S.K., Sa, M., Kwon, J.S., et al. 2020. Immunophenotyping of COVID-19 and influenza highlights the role of type I interferons in development of severe COVID-19. Sci. Immunol. 5, eabd1554.

Lei, X., Dong, X., Ma, R., Wang, W., Xiao, X., Tian, Z., Wang, C., Wang, Y., Li, L., Ren, L., et al. 2020. Activation and evasion of type I interferon responses by SARS-CoV-2. Nat. Commun. 11, 3810.

Li, Q., Guan, X., Wu, P., Wang, X., Zhou, L., Tong, Y., Ren, R., Leung, 
K.S.M., Lau, E.H.Y., Wong, J.Y., et al. 2020a. Early transmission dynamics in Wuhan, China, of novel coronavirus-infected pneumonia. N. Engl. J. Med. 382, 1199-1207.

Li, J.Y., Liao, C.H., Wang, Q., Tan, Y.J., Luo, R., Qiu, Y., and Ge, X.Y. 2020b. The ORF6, ORF8 and nucleocapsid proteins of SARSCoV-2 inhibit type I interferon signaling pathway. Virus Res. 286, 198074.

Li, A., Zhao, K., Zhang, B., Hua, R., Fang, Y., Jiang, W., Zhang, J., Hui, L., Zheng, Y., Li, Y., et al. 2021. SARS-CoV-2 NSP12 protein is not an interferon- $\beta$ antagonist. J. Virol. 95, e0074721.

Lin, X., Fu, B., Yin, S., Li, Z., Liu, H., Zhang, H., Xing, N., Wang, Y., Xue, W., Xiong, Y., et al. 2021. ORF8 contributes to cytokine storm during SARS-CoV-2 infection by activating IL-17 pathway. iScience 24, 102293.

Liu, G., Lee, J.H., Parker, Z.M., Acharya, D., Chiang, J.J., van Gent, M., Riedl, W., Davis-Gardner, M.E., Wies, E., Chiang, C., et al. 2021. ISG15-dependent activation of the sensor MDA5 is antagonized by the SARS-CoV-2 papain-like protease to evade host innate immunity. Nat. Microbiol. 6, 467-478.

Lu, X., Pan, J., Tao, J., and Guo, D. 2011. SARS-CoV nucleocapsid protein antagonizes IFN- $\beta$ response by targeting initial step of IFN- $\beta$ induction pathway, and its C-terminal region is critical for the antagonism. Virus Genes 42, 37-45.

Lu, R., Zhao, X., Li, J., Niu, P., Yang, B., Wu, H., Wang, W., Song, H., Huang, B., Zhu, N., et al. 2020. Genomic characterisation and epidemiology of 2019 novel coronavirus: implications for virus origins and receptor binding. Lancet 395, 565-574.

Miao, G., Zhao, H., Li, Y., Ji, M., Chen, Y., Shi, Y., Bi, Y., Wang, P., and Zhang, H. 2021. ORF3a of the COVID-19 virus SARS-CoV-2 blocks HOPS complex-mediated assembly of the SNARE complex required for autolysosome formation. Dev. Cell 56, 427-442.

Miorin, L., Kehrer, T., Sanchez-Aparicio, M.T., Zhang, K., Cohen, P., Patel, R.S., Cupic, A., Makio, T., Mei, M., Moreno, E., et al. 2020. SARS-CoV-2 Orf6 hijacks Nup98 to block stat nuclear import and antagonize interferon signaling. Proc. Natl. Acad. Sci. USA 117, 28344-28354.

Moustaqil, M., Ollivier, E., Chiu, H.P., Van Tol, S., Rudolffi-Soto, P., Stevens, C., Bhumkar, A., Hunter, D.J.B., Freiberg, A.N., Jacques, D., et al. 2021. SARS-CoV-2 proteases PLpro and 3CLpro cleave IRF3 and critical modulators of inflammatory pathways (NLRP12 and TAB1): Implications for disease presentation across species. Emerg. Microbes Infect. 10, 178-195.

Mu, J., Fang, Y., Yang, Q., Shu, T., Wang, A., Huang, M., Jin, L., Deng, F., Qiu, Y., and Zhou, X. 2020. SARS-CoV-2 N protein antagonizes type I interferon signaling by suppressing phosphorylation and nuclear translocation of STAT1 and STAT2. Cell Discov. 6, 65.

Mukherjee, R., Bhattacharya, A., Bojkova, D., Mehdipour, A.R., Shin, D., Khan, K.S., Cheung, H.H.Y., Wong, K.B., Ng, W.L., Cinatl, J., et al. 2021. Famotidine inhibits toll-like receptor 3-mediated inflammatory signaling in SARS-CoV-2 infection. J. Biol. Chem. 297, 100925.

Oh, S.J. and Shin, O.S. 2021. SARS-CoV-2 nucleocapsid protein targets RIG-I-like receptor pathways to inhibit the induction of interferon response. Cells 10, 530.

Park, A. and Iwasaki, A. 2020. Type I and type III interferons - induction, signaling, evasion, and application to combat COVID-19. Cell Host Microbe 27, 870-878.

Perlman, S. and Netland, J. 2009. Coronaviruses post-SARS: update on replication and pathogenesis. Nat. Rev. Microbiol. 7, 439-450.

Rashid, F., Dzakah, E.E., Wang, H., and Tang, S. 2021. The ORF8 protein of SARS-CoV-2 induced endoplasmic reticulum stress and mediated immune evasion by antagonizing production of interferon beta. Virus Res. 296, 198350.

Rehwinkel, J. and Gack, M.U. 2020. RIG-I-like receptors: Their regulation and roles in RNA sensing. Nat. Rev. Immunol. 20, 537-551.

Reikine, S., Nguyen, J.B., and Modis, Y. 2014. Pattern recognition and signaling mechanisms of RIG-I and MDA5. Front. Immunol.
5,342 .

Ren, Y., Shu, T., Wu, D., Mu, J., Wang, C., Huang, M., Han, Y., Zhang, X.Y., Zhou, W., Qiu, Y., et al. 2020. The ORF3a protein of SARSCoV-2 induces apoptosis in cells. Cell. Mol. Immunol. 17, 881883.

Salvi, V., Nguyen, H.O., Sozio, F., Schioppa, T., Gaudenzi, C., Laffranchi, M., Scapini, P., Passari, M., Barbazza, I., Tiberio, L., et al. 2021. SARS-CoV-2-associated ssRNAs activate inflammation and immunity via TLR7/8. JCI Insight 22, e150542.

Sampaio, N.G., Chauveau, L., Hertzog, J., Bridgeman, A., Fowler, G., Moonen, J.P., Dupont, M., Russell, R.A., Noerenberg, M., and Rehwinkel, J. 2021. The RNA sensor MDA5 detects SARS-CoV-2 infection. Sci. Rep. 11, 13638.

Schneider, W.M., Chevillotte, M.D., and Rice, C.M. 2014. Interferonstimulated genes: a complex web of host defenses. Annu. Rev. Immunol. 32, 513-545.

Schubert, K., Karousis, E.D., Jomaa, A., Scaiola, A., Echeverria, B., Gurzeler, L.A., Leibundgut, M., Thiel, V., Muhlemann, O., and Ban, N. 2020. SARS-CoV-2 Nsp1 binds the ribosomal mRNA channel to inhibit translation. Nat. Struct. Mol. Biol. 27, 959-966.

Shi, C.S., Qi, H.Y., Boularan, C., Huang, N.N., Abu-Asab, M., Shelhamer, J.H., and Kehrl, J.H. 2014. SARS-coronavirus open reading frame-9b suppresses innate immunity by targeting mitochondria and the MAVS/TRAF3/TRAF6 signalosome. J. Immunol. 193, 3080-3089.

Shin, D., Mukherjee, R., Grewe, D., Bojkova, D., Baek, K., Bhattacharya, A., Schulz, L., Widera, M., Mehdipour, A.R., Tascher, G., et al. 2020. Papain-like protease regulates SARS-CoV-2 viral spread and innate immunity. Nature 587, 657-662.

Shirato, K. and Kizaki, T. 2021. SARS-CoV-2 spike protein S1 subunit induces pro-inflammatory responses via toll-like receptor 4 signaling in murine and human macrophages. Heliyon 7, e06187.

Thoms, M., Buschauer, R., Ameismeier, M., Koepke, L., Denk, T., Hirschenberger, M., Kratzat, H., Hayn, M., Mackens-Kiani, T., Cheng, J., et al. 2020. Structural basis for translational shutdown and immune evasion by the Nsp1 protein of SARS-CoV-2. Science 369, 1249-1255.

Thorne, L.G., Reuschl, A.K., Zuliani-Alvarez, L., Whelan, M.V.X., Turner, J., Noursadeghi, M., Jolly, C., and Towers, G.J. 2021. SARS-CoV-2 sensing by RIG-I and MDA5 links epithelial infection to macrophage inflammation. EMBO J. 40, e107826.

Totura, A.L., Whitmore, A., Agnihothram, S., Schäfer, A., Katze, M.G., Heise, M.T., and Baric, R.S. 2015. Toll-like receptor 3 signaling via TRIF contributes to a protective innate immune response to severe acute respiratory syndrome coronavirus infection. mBio 6, e00638-15.

Toyoshima, Y., Nemoto, K., Matsumoto, S., Nakamura, Y., and Kiyotani, K. 2020. SARS-CoV-2 genomic variations associated with mortality rate of COVID-19. J. Hum. Genet. 65, 1075-1082.

Vazquez, C., Swanson, S.E., Negatu, S.G., Dittmar, M., Miller, J., Ramage, H.R., Cherry, S., and Jurado, K.A. 2021. SARS-CoV-2 viral proteins NSP1 and NSP13 inhibit interferon activation through distinct mechanisms. PLoS ONE 16, e0253089.

Wang, S., Dai, T., Qin, Z., Pan, T., Chu, F., Lou, L., Zhang, L., Yang, B., Huang, H., Lu, H., et al. 2021a. Targeting liquid-liquid phase separation of SARS-CoV-2 nucleocapsid protein promotes innate antiviral immunity by elevating MAVS activity. Nat. Cell Biol. 23, 718-732.

Wang, W., Zhou, Z., Xiao, X., Tian, Z., Dong, X., Wang, C., Li, L., Ren, L., Lei, X., Xiang, Z., et al. 2021b. SARS-CoV-2 nsp12 attenuates type I interferon production by inhibiting IRF3 nuclear translocation. Cell. Mol. Immunol. 18, 945-953.

Wu, Z. and McGoogan, J.M. 2020. Characteristics of and important lessons from the coronavirus disease 2019 (COVID-19) outbreak in China: Summary of a report of 72314 cases from the Chinese Center for Disease Control and Prevention. JAMA 323, 12391242 . 
Wu, J., Shi, Y., Pan, X., Wu, S., Hou, R., Zhang, Y., Zhong, T., Tang, H., Du, W., Wang, L., et al. 2021. SARS-CoV-2 ORF9b inhibits RIG-I-MAVS antiviral signaling by interrupting K63-linked ubiquitination of NEMO. Cell Rep. 34, 108761.

Xia, H., Cao, Z., Xie, X., Zhang, X., Chen, J.Y.C., Wang, H., Menachery, V.D., Rajsbaum, R., and Shi, P.Y. 2020. Evasion of type i Interferon by SARS-CoV-2. Cell Rep. 33, 108234.

Yamada, T., Sato, S., Sotoyama, Y., Orba, Y., Sawa, H., Yamauchi, H., Sasaki, M., and Takaoka, A. 2021. RIG-I triggers a signalingabortive anti-SARS-CoV-2 defense in human lung cells. Nat. Immunol. 22, 820-828.

Yang, L., Xie, X., Tu, Z., Fu, J., Xu, D., and Zhou, Y. 2021. The signal pathways and treatment of cytokine storm in COVID-19. Sig. Transduct. Target. Ther. 6, 255.

Yang, Z., Zhang, X., Wang, F., Wang, P., Kuang, E., and Li, X. 2020. Suppression of MDA5-mediated antiviral immune responses by NSP8 of SARS-CoV-2. bioRxiv. doi: https://doi.org/10.1101/2020. 08.12.247767.

Yin, X., Riva, L., Pu, Y., Martin-Sancho, L., Kanamune, J., Yamamoto, Y., Sakai, K., Gotoh, S., Miorin, L., De Jesus, P.D., et al. 2021. MDA5 governs the innate immune response to SARS-CoV-2 in lung epithelial cells. Cell Rep. 34, 108628.

Yuen, C.K., Lam, J.Y., Wong, W.M., Mak, L.F., Wang, X., Chu, H., Cai, J.P., Jin, D.Y., To, K.K.W., Chan, J.F.W., et al. 2020. SARS CoV-2 nsp13, nsp14, nsp15 and orf6 function as potent interferon antagonists. Emerg. Microbes Infect. 9, 1418-1428.

Zhang, Q., Bastard, P., Liu, Z., Le Pen, J., Moncada-Velez, M., Chen, J., Ogishi, M., Sabli, I.K.D., Hodeib, S., Korol, C., et al. 2020. Inborn errors of type I IFN immunity in patients with life-threatening COVID-19. Science 370, eabd4570.

Zhang, Y., Chen, Y., Li, Y., Huang, F., Luo, B., Yuan, Y., Xia, B., Ma, X., Yang, T., Yu, F., et al. 2021a. The ORF8 protein of SARS-CoV2 mediates immune evasion through down-regulating MHC-I. Proc. Natl. Acad. Sci. USA 118, e2024202118.

Zhang, K., Miorin, L., Makio, T., Dehghan, I., Gao, S., Xie, Y., Zhong,
H., Esparza, M., Kehrer, T., Kumar, A., et al. 2021b. Nsp1 protein of SARS-CoV-2 disrupts the mRNA export machinery to inhibit host gene expression. Sci. Adv. 7, eabe7386.

Zhao, Y., Kuang, M., Li, J., Zhu, L., Jia, Z., Guo, X., Hu, Y., Kong, J., Yin, H., Wang, X., et al. 2021a. SARS-CoV-2 spike protein interacts with and activates TLR41. Cell Res. 31, 818-820.

Zhao, Y., Sui, L., Wu, P., Wang, W., Wang, Z., Yu, Y., Hou, Z., Tan, G., Liu, Q., and Wang, G. 2021b. A dual-role of SARS-CoV-2 nucleocapsid protein in regulating innate immune response. Sig. Transduct. Target. Ther. 6, 331.

Zheng, M., Karki, R., Williams, E.P., Yang, D., Fitzpatrick, E., Vogel, P., Jonsson, C.B., and Kanneganti, T.D. 2021. TLR2 senses the SARS-CoV-2 envelope protein to produce inflammatory cytokines. Nat. Immunol. 22, 829-838.

Zheng, Y., Zhuang, M.W., Han, L., Zhang, J., Nan, M.L., Zhan, P., Kang, D., Liu, X., Gao, C., and Wang, P.H. 2020. Severe acute respiratory syndrome coronavirus 2 (SARS-CoV-2) membrane (M) protein inhibits type I and III interferon production by targeting RIG-I/MDA-5 signaling. Sig. Transduct. Target. Ther. 5, 299.

Zhou, P., Yang, X.L., Wang, X.G., Hu, B., Zhang, L., Zhang, W., Si, H.R., Zhu, Y., Li, B., Huang, C.L., et al. 2020. A pneumonia outbreak associated with a new coronavirus of probable bat origin. Nature 579, 270-273.

Zhu, N., Zhang, D., Wang, W., Li, X., Yang, B., Song, J., Zhao, X., Huang, B., Shi, W., Lu, R., et al. 2020. A novel coronavirus from patients with pneumonia in China, 2019. N. Engl. J. Med. 382, 727-733.

Ziegler, C.G.K., Allon, S.J., Nyquist, S.K., Mbano, I.M., Miao, V.N., Tzouanas, C.N., Cao,Y., Yousif, A.S., Bals, J., Hauser, B.M., et al. 2020. SARS-CoV-2 receptor ACE2 is an interferon-stimulated gene in human airway epithelial cells and is detected in specific cell subsets across tissues. Cell 181, 1016-1035.

Zotta, A., Hooftman, A., and O'Neill, L.A.J. 2021. SARS-CoV-2 targets MAVS for immune evasion. Nat. Cell Biol. 23, 682-683. 